\title{
Violencia en el noviazgo y consumo de alcohol y drogas ilegales entre adolescentes y jóvenes españoles
}

\section{Relationship between dating violence and use of alcohol and illegal drugs in Spanish adolescents and young adults} Marina Julia Muñoz-Rivas*; Manuel Gámez-Guadi**;
José Luis Graña*; ${ }^{* *}$ Liria Fernández
*Departamento de Psicología Biológica y de la Salud, Facultad de Psicología, Universidad Autónoma de Madrid, Madrid, España. ** Departamento de Psicología Clínica, Facultad de Psicología, Universidad Complutense de Madrid, Madrid, España.

Enviar correspondencia a:

Marina J. Muñoz-Rivas. Departamento de Psicología Biológica y de la Salud, Facultad de Psicología, Universidad Autónoma de Madrid, 28049 Madrid, España. Marina.munoz@uam.es.

Fax: 00-34-91.497.52.15. Telf.: 914978751

\section{RESUMEN}

El objetivo de este estudio fue examinar la relación entre consumo de sustancias (i.e., alcohol y drogas ilegales) y violencia en parejas de adolescentes y jóvenes. La muestra estuvo compuesta por 1.282 participantes entre 15 y 20 años. A través de análisis de conglomerados se identificaron tres grupos de adolescentes y jóvenes con diferentes niveles de consumo de sustancias: bajo, moderado y elevado consumo de alcohol y drogas ilegales. Se efectuaron varios análisis de regresión logística para pronosticar cada tipo de agresión contra la pareja (fisica, psicológica y sexual) a partir del nivel de uso de sustancias. Niveles elevados de consumo de alcohol y drogas ilegales incrementaron significativamente la probabilidad de informar de agresión física y sexual para ambos sexos. Asi mismo, los participantes con un nivel elevado de consumo informaron con mayor frecuencia que eran ellos quienes iniciaban los episodios de agresión contra su pareja. Los resultados sugieren que el uso de alcohol y drogas constituye un factor de riesgo para la violencia en el noviazgo, hecho que ha de ser tenido en cuenta para la prevención de la violencia en la pareja ya desde la adolescencia.

Palabras clave: violencia en el noviazgo, consumo de alcohol, drogas ilegales, agresión sexual, adolescencia, conductas de riesgo.
Key words: dating aggression, alcohol use, illegal drugs, sexual aggression, adolescence, risk behaviors. 
a violencia que tiene lugar en las relaciones de noviazgo representa un serio problema social que conlleva importantes consecuencias a corto y largo plazo para la salud de las víctimas (Ackard y Neumark-Sztainer, 2002; Kaura y Lohman, 2007; Silverman, Raj, Mucci y Hathaway, 2001). De acuerdo con los estudios disponibles, hasta un $50 \%$ de jóvenes han ejercido alguna forma de agresión física contra su pareja (Cascardi, Avery-Leaf, O'Leary y Slep, 1999; Foshee, 1996; O'Leary y Cascardi, 1998; O'Leary, Slep, Avery-Leaf y Cascardi, 2008) y un porcentaje aún mayor informa haber empleado agresión psicológica (MuñozRivas, Graña, O'Leary y González, 2007; Schumacher y Slep, 2004; Shook, Gerrity, Jurich y Segrist, 2000; White, Merrill y Koss, 2001). Asimismo, la tasa de jóvenes que informan haber perpetrado agresión sexual alcanza el 37\% para los varones y el $24 \%$ para las mujeres (Hickman, Jaycox y Aronoff, 2004; Hines y Saudino, 2003; Muñoz-Rivas, Graña, O'Leary y González, 2009).

Un buen número de relaciones de noviazgo se inician en la adolescencia, etapa en la cual es habitual el uso de alcohol y drogas ilegales (Calvete y Estévez, 2009; Feldstein y Miller, 2006; Jessor, 1987; Jiménez-Muro, Beamonte, Marqueta, Gargallo, Nerín, 2009). Entre adolescentes españoles, el $80 \%$ ha consumido alcohol en al menos una ocasión, el $35 \%$ cannabis y entre el $1 \%$ y el $6 \%$ ha usado alguna vez cocaína, alucinógenos, éxtasis, anfetaminas o heroína según la Encuesta Estatal sobre Uso de Drogas en Estudiantes de Enseñanzas Secundarias (Estudes, 2008). Además, en esta etapa el policonsumo de sustancias es frecuente y conlleva importantes consecuencias. En torno al 90\% de los adolescentes que han tomado éxtasis, alucinógenos, anfetaminas o cocaina han consumido también cannabis o alcohol, y un porcentaje superior al 95\% de los que han consumido en alguna ocasión cannabis también han ingerido alcohol (Estudes, 2008).

El consumo de sustancias constituye una práctica común entre adolescentes y jóvenes que con frecuencia aparece relacionada con otras conductas de riesgo como el empleo de violencia en las relaciones de pareja. En este sentido, las investigaciones llevadas a cabo con muestras anglosajonas han hallado que el consumo de sustancias incrementa la probabilidad, tanto en varones como en mujeres adolescentes, de emplear diferentes tipos de agresión en sus relaciones de noviazgo (Eaton, Davis, Barrios, Brener y Noonan, 2007; Harrison, Erickson, Adlaf y Freeman, 2001; Howard, Wang y Yan, 2007; Huang, White, Kosterman, Catalano y Hawkins, 2001; Kuntsche, Knibbe, Engels y Gmel, 2007; Lacasse y Mendelson, 2007; MacDonald, Piquero, Valois y Zullig, 2005; Swahn, Bossarte y Sullivent, 2008).

Diversos modelos teóricos han sido propuestos para explicar la relación entre consumo de sustancias y violencia. Por ejemplo, se ha señalado que el alcohol y otras drogas podrian incrementar la agresión a través de su efecto psicofarmacológico directo sobre los procesos atencionales, interpretativos o de toma de decisiones (Abbey, Zawacki y McAuslan, 2000; Ito, Miller y Pollock, 1996). De forma complementaria a las explicaciones basadas en el efecto farmacológico directo, un buen número de trabajos sugiere la existencia de una relación indirecta entre consumo de sustancias y violencia (Abbey, Zawacki, Buck, Clinton y McAuslan, 2004; Champion et al., 2004). Así, de acuerdo con la Teoría del Comportamiento Problema (Jessor, 1987, 1991; Jessor et al., 2003), los adolescentes y jóvenes que se implican en un comportamiento de riesgo para la salud es probable que también lleven a cabo otros, aunque no exista necesariamente una relación causal entre ellos (Feldstein y Miller, 2006; Quigley y Leonard, 2000). En este sentido, numerosos estudios han informado de la existencia de un sindrome de comportamientos problemáticos o de riesgo, dentro del cual el consumo de sustancias y la violencia habitualmente covarían (DuRant et al., 2007; Eaton et al., 2007; Howard et al., 2007; King et al., 2004) y podrian compartir el mismo mecanismo explicativo subyacente (Jessor, 1993; Peña, Andreu y Graña, 2008).

La mayor parte de la investigación sobre la relación entre violencia en el noviazgo y consumo de sustancias ha sido llevada a cabo con muestras anglosajonas, siendo escasa la evidencia empírica al respecto en muestras hispanohablantes. Sin embargo, algunos hallazgos sugieren que esta relación podría diferir entre distintos contextos nacionales. Por ejemplo, se ha encontrado que la asociación entre varios comportamientos de riesgo, como el abuso de alcohol, el consumo de otras drogas y la violencia, varía en función del entorno cultural dependiendo de las características de cada país (Eisner, 2002) y, más específicamente, se ha informado que la relación entre consumo de sustancias y agresión en el noviazgo difiere entre diversos contextos nacionales (Hines y Straus, 2007). Por otra parte, a pesar de que el poli-consumo de sustancias es frecuente en adolescentes, son pocos los estudios que han investigado la asociación entre la violencia en el noviazgo y patrones de consumo que incluyan tanto alcohol como drogas ilegales.

Por tanto, el objetivo de este estudio fue analizar la relación entre diferentes niveles de consumo de alcohol y drogas ilegales y agresión física, psicológica y sexual en parejas de adolescentes y jóvenes españoles. De acuerdo con la evidencia empírica arriba revisada, hipotetizamos que niveles mayores de consumo de sustancias estarán asociados con la perpetración de agresión física, psicológica y sexual en relaciones de noviazgo para ambos sexos. Asimismo, dado que con frecuencia se ha señalado que la investigación sobre violencia en la pareja ha desatendido el estudio de diferentes variables contextuales de la agresión (Dobash y Dobash, 2004), un objetivo adicional de este trabajo fue explorar si el consumo de sustancias está relacionado con los motivos de los jóvenes para llevar a cabo la conducta agresiva. 


\section{MÉTODO}

\section{Participantes}

En función de los objetivos del estudio, se establecieron los siguientes criterios de inclusión y exclusión:

- Criterios de inclusión: a) tener una edad comprendida entre los 15 y los 20 años, y b) estar en una relación de noviazgo heterosexual en el momento de la evaluación. Las relaciones de pareja del mismo sexo no fueron incluidas en los análisis debido a su reducido número en la muestra.

- Criterio de exclusión: estar casado.

Para la obtención de la muestra se efectuó una selección al azar de 36 centros educativos de la Comunidad de Madrid en los que se cursaban estudios preuniversitarios, de los cuales 20 centros públicos accedieron a participar en el estudio. Los objetivos y el procedimiento de la investigación fueron explicados a los responsables de cada centro, y de igual forma se procedió con las Asociaciones de Padres de Alumnos (APA). La participación de los adolescentes fue anónima y voluntaria, y menos del 1\% rechazó completar el cuestionario.

La muestra total recogida fue de 3.165 adolescentes y jóvenes. Después de eliminar los casos que no cumplían los criterios de inclusión y exclusión, la muestra quedó compuesta por 1.282 adolescentes y jóvenes (56,1\% mujeres y $43,9 \%$ varones) con una edad media de 17,2 años ( $D T=$ $1,30)$, que estaban actualmente manteniendo una relación de noviazgo. El 62\% de los participantes describió su relación como estable o seria y el 35\% como nueva o casual. Respecto a la frecuencia de contacto, el 14\% de los adolescentes veía a su pareja una vez a la semana o menos, el $34 \%$ varias veces a la semana, el $41 \%$ cada día y el $11 \%$ más de una vez al día.

\section{Medidas y Variables}

Se aplicaron los siguientes instrumentos de evaluación:

a) Cuestionario Sociodemográfico. Se incluyeron preguntas relativas al sexo, la edad, el curso académico, el lugar de nacimiento y la orientación sexual. Asimismo, se recogió información referida a las relaciones de pareja mantenidas y a la relación de pareja actual: edad de la primera relación, tipo de relaciones y duración de las mismas.

b) Versión Modificada de la Escala de Tácticas para los Conflictos (M-CTS; Cascardi et al., 1999). La M-CTS está compuesta por 18 ítems con un formato de respuesta de 5 puntos tipo Likert desde 1 (Nunca) hasta 5 (Muy a menudo) que evalúan la presencia de negociación, agresión física y agresión verbal en la relación actual o más reciente. Esta escala ha sido validada recientemente en una muestra española (Muñoz-Rivas,
Andreu, Graña, O'Leary y González, 2007). En base a los objetivos de este estudio, se emplearon las subescalas de agresión verbal ( 5 ítems; á $=0,66)$ y agresión física (10 ítems; á $=0,78)$.

c) Escala de Agresión Sexual entre Adolescentes y Jóvenes. Se elaboró una escala compuesta por 5 ítems para medir la presencia de los comportamientos de agresión sexual más frecuentes que tienen lugar en las relaciones de noviazgo. Este instrumento incluyó cuestiones relativas a presión o coacción para obligar a la pareja a mantener relaciones sexuales no consentidas (p.ej., "¿Tú has amenazado a tu novio/a con terminar la relación si no mantenía relaciones sexuales contigo?", "¿Tú has agarrado o sujetado a tu novio/a para realizar prácticas sexuales que él/ella no quería consentir?), con un formato de respuesta tipo Likert desde 1 (nunca) hasta 5 (muy a menudo). Los ítems fueron elaborados en base a una revisión de los ítems incluidos en instrumentos previos (p.ej., CTS2; Straus, Hamby, Boney-McCoy y Sugarman, 1996). Su consistencia interna fue de á $=0,77$. Se realizó una análisis factorial exploratorio para analizar la estructura interna de este instrumento, obteniéndose un único factor en el que todos los items presentaron cargas factoriales superiores a 0,30.

d) Justificación de la agresión. Se elaboró un ítem para que los participantes que habían informado de algún tipo de agresión física contra su pareja indicasen los motivos a los que atribuian tal agresión. El contenido del ítem fue "¿Por qué motivos has agarrado, empujado, abofeteado, golpeado, etc., a tu novio/a?" con seis alternativas de respuesta: (a) estaba celoso; (b) estaba furioso con él/ ella y golpeé primero; (c) mi novio/a me pegó primero y yo respondí; (d) la agresión se produjo como un juego o una broma; (e) la agresión se produjo en medio de una discusión; y (f) ataqué a mi pareja porque yo estaba nervioso/a.

e) Consumo de sustancias. El consumo de alcohol y drogas ilegales fue evaluado empleando cuestiones con un formato similar al de los índices internacionales estandarizados más empleados para evaluar el uso de sustancias entre adolescentes (p.ej., Youth Risk Behavior Survey).

- Consumo de Alcohol. La ingesta de alcohol fue medida por una escala compuesta por los siguientes tres ítems: (1) "A lo largo de tu vida, ¿cuántos dias has tomado al menos una copa de alcohol?" [Formato de respuesta de 7 alternativas desde 1(Cero días) hasta 7 (100 o más días)]; (2) "Durante los últimos 30 días, ¿cuántos días has tomado al menos una copa de alcohol?; y (3) "Durante los últimos 30 días, ¿cuántos días has tomado al menos una vez alcohol dentro del centro de enseñanza?". El formato de respuesta para los ítems 2 y 3 estuvo compuesto por 7 alternativas desde 1 (Cero días) hasta 7 (Todos los días). La consistencia interna de esta escala fue 
de $\alpha=0,60$. Todos los ítems presentaron una carga factorial en un mismo factor superior a 0,40.

- Consumo de drogas ilegales. Para evaluar el uso de sustancias ilegales se muestreó el consumo de marihuana y el consumo de anfetaminas empleando una escala compuesta por seis cuestiones: (1) "A lo largo de tu vida, ¿cuántas veces has fumado al menos una vez hachís o marihuana?"; (2) "A lo largo de tu vida, ¿cuántas veces has consumido anfetaminas?"; (3) "Durante los últimos 30 días, ¿con qué frecuencia has fumado marihuana o hachis?"; (4) "Durante los últimos 30 días, ¿con qué frecuencia has consumido anfetaminas?"; (5) "Durante los últimos 30 dias, ¿cuántas veces has fumado hachís o marihuana en tu centro de enseñanza?"; y (6) "Durante los últimos 30 dias, ¿cuántas veces has consumido anfetaminas en tu centro de enseñanza?". El formato de respuesta fue de seis alternativas desde 1 (Cero veces) hasta 6 (Cuarenta o más veces). La consistencia interna de esta escala fue de $\alpha=0,71$. Todos los ítems presentaron cargas superiores a 0,50 en el mismo factor.

f) Deseabilidad social. Para controlar la tendencia de algunos participantes a minimizar la revelación de comportamientos socialmente indeseables se empleó una versión adaptada a adolescentes de 25 ítems de la Escala de Deseabilidad Social de Marlowe y Crowne (Crowne y Marlowe, 1960), validada en castellano por Ferrando y Chico (2000). Esta escala mide la tendencia a responder de un modo socialmente aceptable. Incluye ítems directos (p.ej., "Siempre soy cortés, incluso con la gente desagradable") e inversos (p.ej., "A veces trato de vengarme en lugar de perdonar y olvidar lo que me han hecho"), con un formato de respuesta de cuatro alternativas tipo Likert en la que los participantes han de indicar su grado de acuerdo con cada afirmación. La suma de todos los ítems, tras recodificar los inversos, constituye la puntuación global de la escala. Su consistencia interna para este estudio fue de $\alpha=0,78$.

\section{Análisis de datos}

Con el objetivo de identificar grupos homogéneos de adolescentes con diferentes niveles de consumo de sustancias que incluyesen tanto alcohol como drogas ilegales, se efectuó un análisis de conglomerados con las variables "consumo de alcohol" y "consumo de drogas ilegales" como variables de agrupación. El análisis de conglomerados constituye un conjunto de técnicas usadas para identificar grupos homogéneos de casos o variables basados en unas determinadas características. Los casos son agrupados en los conglomerados con el objetivo de minimizar la variabilidad dentro de cada grupo y maximizar las diferencias entre grupos en las variables de agrupación.

En primer lugar, se llevaron a cabo varios análisis de conglomerados jerárquicos con diferentes submuestras aleatorias de casos y dos métodos de conglomeración (Vinculación Intergrupos y Método de Ward), con el objetivo de explorar el número más apropiado de conglomerados y la estabilidad de distintas soluciones a través de diferentes métodos y muestras. Las variables de agrupación fueron estandarizadas en puntuaciones $Z$ para que compartiesen una métrica común y su contribución fuese equiparable. Se examinaron las soluciones de conglomerados entre 2 y 6 grupos. Finalmente, se optó por una solución en tres conglomerados porque resultó ser la más estable e interpretable. En una segunda fase, un análisis de conglomerados $\mathrm{K}$ medias fue llevado a cabo para la muestra al completo especificando una solución en tres conglomerados. A continuación, con el objetivo de definir cada conglomerado e identificar sus características, se efectuó un Análisis de Varianza (ANOVA) para comparar los conglomerados obtenidos en las variables empleadas para la agrupación y en el resto de variables analizadas. Adicionalmente, se llevó a cabo un análisis discriminante para confirmar la solución de conglomerados obtenida. Para pronosticar la perpetración de agresión a partir del nivel de consumo, se efectuaron una serie de análisis de regresión logística con el tipo de agresión (física, psicológica o sexual) como variable dependiente, controlando el efecto de la edad, la duración de la relación y la deseabilidad social. Cada tipo de agresión fue dicotomizado y codificado como 0 (ausencia de agresión) y 1 (agresión en alguna ocasión). Finalmente, las diferencias entre los distintos niveles de consumo en cuanto a los motivos para justificar la agresión fueron analizadas empleando la prueba Chi-cuadrado de Pearson. Los análisis estadísticos fueron llevados a cabo con el paquete estadístico SPSS 16.

\section{RESULTADOS}

\section{Caracteristicas descriptivas de la muestra}

De la muestra total de adolescentes, el $89 \%$ de los varones y el $88 \%$ de las mujeres habían ingerido alcohol en algún momento de su vida $\left[\chi_{(1)}^{2}=0,08 ; p=0,77\right]$, el $72,1 \%$ y el $67,7 \%$ de varones y mujeres respectivamente lo ingirieron durante los últimos 30 dias $\left[\chi_{(1)}^{2}=2,63 ; p=\right.$ $0,10]$ y el $10,5 \%$ de los varones y el $6,1 \%$ de las mujeres lo consumieron el último mes en su centro de enseñanza $\left[\chi^{2}\right.$ (1) $=8,24 ; p<0,05]$. Respecto al consumo de drogas ilegales, el $57,3 \%$ de los varones y el $52,4 \%$ de las mujeres $\left[\chi^{2}\right.$ (1) $=3,12 ; p=0,07$ ] informaron haber usado marihuana en alguna ocasión, el $32,8 \%$ de los varones y el $27,8 \%$ de las mujeres la usaron durante los últimos 30 días $\left[\chi_{(1)}^{2}=3,71\right.$; $p=0,06]$, y el $12,9 \%$ de los varones y el $8,7 \%$ y de las mujeres la usaron el mes pasado en el centro de enseñanza $\left[\chi^{2}{ }_{(1)}=5,79 ; p<0,01\right]$. Además, el $9,7 \%$ de los varones $y$ el $9,1 \%$ de las mujeres $\left[\chi_{(1)}^{2}=0,69 ; p=0,14\right]$ consumieron anfetaminas alguna vez en su vida, el 3,3\% de los hombres y el $1,1 \%$ de las mujeres lo hicieron durante los últimos 30 
dias $\left[\chi^{2}{ }_{(1)}=6,82 ; p<0,01\right]$, y el $1,6 \%$ de los varones $y$ el $0,1 \%$ de las mujeres las consumieron el último mes en su centro de enseñanza $\left[\chi_{(1)}^{2}=8,68 ; p<0,01\right]$. Respecto a la prevalencia de los diferentes tipos de agresión, el 37\% de los varones y el $46 \%$ de las mujeres ejercieron agresión física en algún momento $\left[\chi_{(1)}^{2}=11,07 ; p<0,01\right]$, el $90 \%$ de los varones y el $94,8 \%$ de las mujeres $\left[\chi_{(1)}^{2}=11,89 ; p\right.$ $=0,01]$ se implicaron en algún tipo de agresión psicológica contra su actual pareja, y el $26 \%$ de los varones y el $9,8 \%$ de las mujeres $\left[\chi_{(1)}^{2}=60,45 ; p<0,001\right]$ emplearon algún tipo de coacción sexual en el contexto de sus relaciones de noviazgo.

\section{Niveles de consumo de sustancias en adolescentes}

En la Tabla 1 se presentan los conglomerados de adolescentes con diferentes niveles de consumo de sustancias y sus puntuaciones en las variables demográficas, de consumo de sustancias y de violencia contra la pareja. El primer conglomerado ( $n=487$ ), compuesto en su mayor parte por mujeres (61\%), presentó niveles de uso de alcohol y drogas ilegales significativamente inferiores a los del resto de conglomerados. Este conglomerado fue denominado grupo con niveles bajos de consumo de alcohol y drogas ilegales. El segundo conglomerado ( $n=540)$ estuvo también integrado por mayor número de mujeres (58\%). El uso promedio de alcohol y drogas ilegales de los adolescentes en este conglomerado fue mayor que el de los adolescentes con bajos niveles de consumo de sustancias (Conglomerado 1), pero inferior al de los participantes en el tercer conglomerado, razón por la cual fue denominado grupo con niveles intermedios de consumo de sustancias. Por último, el tercer conglomerado ( $n=255 ; 52 \%$ hombres) estuvo integrado por adolescentes con niveles elevados de consumo de alcohol y drogas ilegales, que fueron significativamente superiores al del resto de conglomerados (véase Tabla 1). Adicionalmente, los adolescentes con un nivel más elevado de consumo (Conglomerado 3) presentaron puntuaciones significativamente mayores en cada tipo de agresión analizada (física, psicológica y sexual) que los adolescentes con niveles bajos e intermedios de uso de sustancias, que no se diferenciaron entre sí (Tabla 1).

La solución en tres conglomerados obtenida fue confirmada a través de análisis discriminante empleando el conglomerado de pertenencia como variable de clasificación y las variables de consumo de alcohol y drogas ilegales como variables independientes. El 99,5\% de todos los casos agrupados en el análisis de conglomerados K-medias fueron identificados correctamente en el análisis discriminante. Más específicamente, la tasa de identificación correcta fue del 99,8\% en el primer conglomerado, del 99\% para el segundo conglomerado y del $100 \%$ para los casos agrupados en el tercer conglomerado.

Tabla 1. Diferencias entre los conglomerados en las variables sociodemográficas, en el consumo de sustancias y en la violencia en la pareja.

\begin{tabular}{|c|c|c|c|c|}
\hline & $\begin{array}{l}\text { Nivel bajo de consumo de alco- } \\
\text { hol y drogas ilegales. } \mathrm{N}=487 \\
\text { (Conglomerado 1) }\end{array}$ & $\begin{array}{l}\text { Nivel intermedio de consumo de } \\
\text { alcohol y drogas ilegales. } \\
N=540 \text { (Conglomerado 2) }\end{array}$ & $\begin{array}{l}\text { Nivel elevado de consumo de } \\
\text { alcohol drogas ilegales. } \mathrm{N}=255 \\
\text { (Conglomerado } 3 \text { ) }\end{array}$ & $\mathrm{F}$ \\
\hline Sexo $(1=$ mujer $)$ & $0,61 c$ & 0,58 & $0,48 a$ & $5,94^{* *}$ \\
\hline Edad & $16,90 \mathrm{bc}$ & $17,40 a$ & $17,62 a$ & $32,36^{* * *}$ \\
\hline Duración de la relación (en meses) & 10,23 & 11,56 & 11,67 & 0,93 ns \\
\hline \multicolumn{5}{|l|}{ Consumo de sustancias } \\
\hline Alcohol & $-1,05 b c$ & $0,51 \mathrm{ac}$ & $0,92 \mathrm{ac}$ & $1941,52^{* * *}$ \\
\hline Drogas llegales & $-0,58 b c$ & $-0,25 a c$ & $1,63 a b$ & $1951,01^{* * *}$ \\
\hline Marihuana & $-0,60 b c$ & $-0,25 a c$ & $1,65 a b$ & $92,51^{* * *}$ \\
\hline Anfetaminas & $-0,20 c$ & $-0,15 c$ & $0,63 a b$ & $16,49^{* * *}$ \\
\hline \multicolumn{5}{|l|}{ Violencia en la pareja } \\
\hline Agresión Psicológica & $-0,14 c$ & $-0,01 c$ & $0,28 a b$ & $11,82^{* * *}$ \\
\hline Agresión Fisica & $-0,06 c$ & $-0,13 c$ & $0,19 a b$ & $11,81^{* * *}$ \\
\hline Agresión Sexual & $-0,11 c$ & $-0,07 c$ & $0,20 a b$ & $13,67^{* * *}$ \\
\hline
\end{tabular}

Nota. Las puntuaciones en las variables de consumo y violencia han sido previamente estandarizadas

${ }^{* *} p<.01 ;{ }^{* * *} p<0.001$ (Scheffé-test); ns = 'no significativo'.

a'Media significativamente diferente a la del Conglomerado

${ }^{b}$ Media significativamente diferente a la del Conglomerado2

'Media significativamente diferente a la del Conglomerado3 


\section{Predicción de la violencia}

Se llevaron a cabo una serie de análisis de regresión logística para predecir la probabilidad de ejercer cada tipo de agresión a partir del nivel de ingesta de alcohol y drogas ilegales. La variable categórica "nivel de consumo" de alcohol y drogas ilegales ("1= Bajo", "2= Intermedio", "3= Elevado") resultante del análisis de conglomerados fue empleada como variable predictora, controlando el efecto de la edad, la duración de la relación y la deseabilidad social. El grupo con niveles bajos de consumo fue empleado como la categoría de referencia, con la cual se compararon el resto de grupos de consumo (Field, 2005). El tipo de agresión fue dicotomizado ( $0=$ ausencia; $1=$ presencia) y empleado como variable dependiente.

En la Tabla 2 se muestran los resultados de los análisis de regresión en odds ratio (OR). Para la agresión física, la probabilidad de agresión de los participantes con niveles intermedios de consumo no difirió significativamente de la probabilidad de agresión de los participantes con un nivel bajo de consumo de sustancias $(p>0,05)$. Sin embargo, pertenecer al grupo con niveles elevados de consumo de alcohol y drogas sí incrementó significativamente, tanto para los varones como para las mujeres, el riesgo de informar de agresión física contra la pareja $(O R=2,44$ y 2,73 para varones y mujeres, respectivamente). Para analizar con más detalle la relación entre el nivel de consumo y la probabilidad de agresión física, se elaboró un diagrama de efectos condicionales para la regresión logística (Hamilton, 1992), en el que se muestra la probabilidad de perpetrar agresión física en los niveles de bajo y elevado consumo (ver Figura 1), manteniendo constantes en sus puntuaciones medias el resto de variables control mostradas en la Tabla 2. Como se observa en la Figura 1, la probabilidad de agresión física se elevó, en el caso de los hombres, desde 0,30 en el nivel de bajo uso de sustancias hasta 0,50 en el nivel de elevado uso de sustancias. Para las mujeres, la probabilidad aumentó desde 0,40, para aquellas con niveles bajos de consumo, hasta 0,65 para las mujeres con un nivel más elevado de consumo de alcohol y drogas ilegales.

Tabla 2. Análisis de la regresión logística para predecir la violencia en el noviazgo a partir del nivel de consumo de alcohol y drogas ilegales.

\begin{tabular}{|c|c|c|c|}
\hline \multirow[b]{2}{*}{ Variables Independientes } & \multicolumn{3}{|c|}{ Odds Ratio ${ }^{+}$} \\
\hline & $\begin{array}{l}\text { Agresión } \\
\text { Fisica }\end{array}$ & $\begin{array}{c}\text { Agresión } \\
\text { Psicológica }\end{array}$ & $\begin{array}{l}\text { Agresión } \\
\text { sexual }\end{array}$ \\
\hline & \multicolumn{3}{|c|}{ Varones } \\
\hline \multicolumn{4}{|l|}{ Nivel de Consumo } \\
\hline Bajo vs Intermedio & 0,73 ns & 1,10 ns & $2,45^{* *}$ \\
\hline $\begin{array}{l}\text { Bajo vs Alto } \\
\quad \text { Variables Control }\end{array}$ & $2,44^{* *}$ & 2,03 ns & $2,74^{* *}$ \\
\hline Edad & $0,71^{* * *}$ & 0,78 ns & $1,06 n s$ \\
\hline Duración de la relación & $1,04^{* *}$ & $1,07^{* *}$ & 1,00 ns \\
\hline Deseabilidad Social & $0,95^{*}$ & $0,86^{* * *}$ & $0,93^{* * *}$ \\
\hline & \multicolumn{3}{|c|}{ Mujeres } \\
\hline \multicolumn{4}{|l|}{ Nivel de consumo } \\
\hline Bajo vs Intermedio & 1,08 ns & $1,76 n s$ & 1,20 ns \\
\hline $\begin{array}{l}\text { Bajo vs Alto } \\
\quad \text { Variables Control }\end{array}$ & $2,73^{* * *}$ & $2,20 \mathrm{~ns}$ & 3,03 * \\
\hline Edad & 0,92 ns & $0,74 n s$ & $1,23 \mathrm{~ns}$ \\
\hline Duración de la relación & 1,04 ns & 1,03 ns & $1,02^{*}$ \\
\hline Deseabilidad Social & $0,95^{* *}$ & 0,87 & $0,97^{* *}$ \\
\hline
\end{tabular}

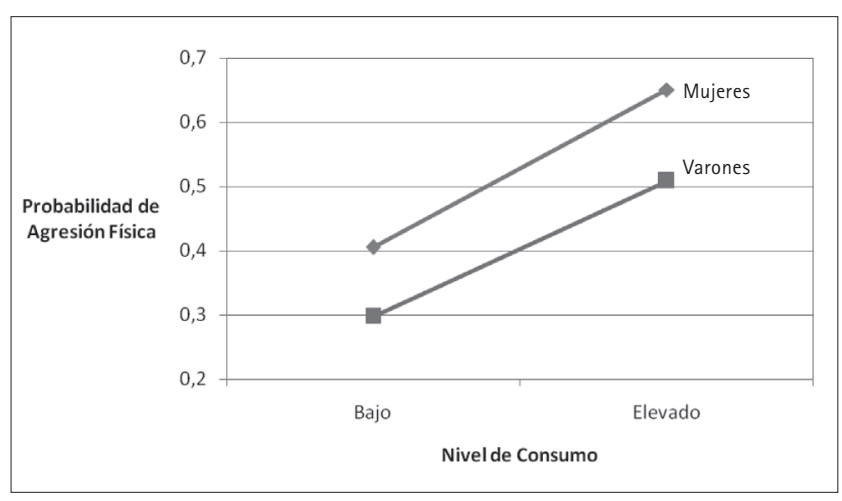

Figura 1. Probabilidad de agresión física en los niveles de bajo y elevado consumo de alcohol y drogas ilegales.

En relación a la agresión psicológica, como se observa en la Tabla 2, la probabilidad de este tipo de agresión en los grupos de intermedio y elevado consumo de sustancias no difirió significativamente de la probabilidad de agresión psicológica entre los participantes en el grupo con niveles más bajos de consumo $(p>0,05)$, ni para los varones ni para mujeres, después de controlar el efecto de la edad, la duración de la relación y la deseabilidad social.

La agresión sexual, por su parte, fue significativamente más probable entre los adolescentes con niveles elevados de consumo. Concretamente, para los hombres las odds ratios de perpetrar agresión sexual contra las mujeres fueron de 2,45 y 2,74 en los niveles de intermedio y elevado consumo, respectivamente. Asimismo, las odds ratio para este tipo de agresión entre las mujeres con niveles elevados de uso de sustancias fue hasta tres veces mayor que entre las adolescentes con niveles bajos de consumo de alcohol y drogas ilegales (Tabla 2). Este incremento se muestra en el diagrama de efectos condicionales para la agresión sexual (véase Figura 2). La probabilidad de agresión sexual se elevó desde 0,23 para los varones con niveles más bajos de consumo, hasta 0,45 en el nivel de consumo elevado. En el caso de las mujeres, la probabilidad de agresión sexual fue menor que para los hombres, aunque también aumentó desde 0,07 para el grupo con un consumo de sustancias más bajo, hasta 0,21 para las adolescentes con un patrón de consumo más elevado.

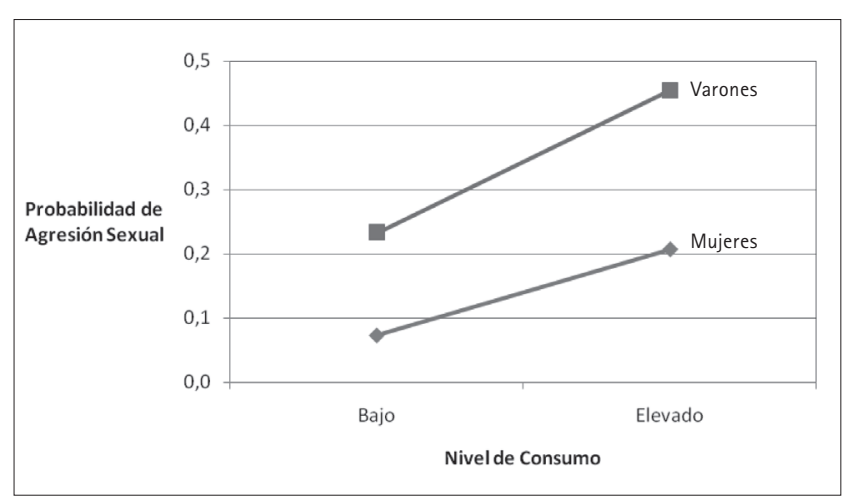

Figura 2. Probabilidad de agresión sexual en los niveles de bajo y elevado consumo de alcohol y drogas ilegales 
Tabla 3. Diferencias entre los conglomerados en los motivos para justificar la agresión

\begin{tabular}{|c|c|c|c|c|}
\hline & $\begin{array}{l}\text { Nivel bajo de consumo de sus- } \\
\text { tancias }\end{array}$ & $\begin{array}{c}\text { Nivel intermedio } \\
\text { de consumo de sustancias }\end{array}$ & $\begin{array}{l}\text { Nivel elevado de consumo de } \\
\text { sustancias }\end{array}$ & $\mathrm{F}$ \\
\hline \multicolumn{5}{|l|}{ Motivos } \\
\hline Estaba celoso & $5,1 \%$ & $5,6 \%$ & $5,1 \%$ & $2,69 n s$ \\
\hline Estaba furioso y le golpeé primero & $2,7 \%$ & $3,3 \%$ & $7,5 \%$ & $11,01^{* *}$ \\
\hline Mi novio me pegó primero y yo respondí & $1,6 \%$ & $1,3 \%$ & $0,8 \%$ & $0,62 n s$ \\
\hline Estaba bromeando & $7,2 \%$ & $5,6 \%$ & $8,6 \%$ & $0,24 n s$ \\
\hline Estábamos discutiendo & $0,4 \%$ & $0,2 \%$ & $0,4 \%$ & $0,78 n s$ \\
\hline Estaba nervioso & $0,8 \%$ & $0,8 \%$ & $3,9 \%$ & $14,58^{* *}$ \\
\hline
\end{tabular}

${ }^{*} p<0,5 ;{ }^{* *} p<0,01 ; n s=$ no significativo.

Respecto a las variables control, la edad resultó significativa únicamente para los varones disminuyendo la probabilidad de agresión física. La duración de la relación, por su parte, aumentó la probabilidad de la agresión física y psicológica para los varones y de la sexual para las mujeres. En el caso de la deseabilidad social, elevadas puntuaciones en esta variable se asociaron, en la mayoría de los casos, con menores niveles de agresión (véase Tabla 2).

\section{Consumo de sustancias y justificación de la agresión}

Por último, se examinó la relación entre el nivel de ingesta de sustancias y la justificación de la agresión (véase Tabla 3).

Como puede observarse en la Tabla 3, un 7,5\% de los adolescentes clasificados en el grupo de elevado consumo informó que ellos habian sido quienes habian iniciado los episodios de violencia, frente al $2,7 \%$ y $3,3 \%$ de los grupos de bajo e intermedio consumo $\left[X^{2}{ }_{(2)}=11,01 ; p<0,01\right]$. Además, el $3,9 \%$ de los adolescentes en el grupo de elevado consumo agredió a su pareja porque estaba nervioso, frente al 0,8\% en cada uno de los otros dos grupos que lo hicieron por este motivo $\left[X_{(2)}^{2}=14,58 ; p<0,01\right]$.

\section{DISCUSIÓN}

El objetivo principal del presente estudio fue el de examinar la relación entre distintos niveles de consumo de sustancias y la perpetración de agresión física, psicológica y sexual en las relaciones de noviazgo de adolescentes españoles. Los resultados sugieren que niveles mayores de consumo de alcohol y drogas ilegales incrementan la probabilidad de ejercer agresión física y sexual contra la pareja, aunque no agresión psicológica. Asimismo, los adolescentes con un nivel más elevado de uso de sustancias informaron que eran ellos quienes iniciaban con mayor frecuencia los episodios violentos.

Un elevado porcentaje de adolescentes informó que había usado sustancias en alguna ocasión o durante el último mes en tasas comparables a las de otros trabajos (Estudes, 2008). Igualmente, un número considerable de adolescentes reconoció haber ejercido algún tipo de violencia contra su pareja, de forma equiparable a lo encontrado por otros estudios (Foshee, 1996; Poitras y Lavoie, 1995).

El análisis de conglomerados identificó tres grupos de adolescentes con distintos niveles (bajo, intermedio y elevado) de ingesta de alcohol y drogas ilegales. El nivel de consumo de sustancias incrementó significativamente la probabilidad de perpetrar agresión física o sexual. Concretamente, un nivel elevado de consumo aumentó entre dos y tres veces el riesgo de agresión física o sexual tanto para varones como para mujeres. Globalmente, estos resultados son congruentes con los resultados de un buen número de investigaciones previas llevadas a cabo con muestras anglosajonas en las que se ha encontrado de forma consistente una relación entre un mayor consumo de sustancias y una mayor probabilidad de usar violencia en las relaciones de noviazgo que tienen lugar durante la adolescencia (Foshee, Linder, MacDougall y Bangdiwala, 2001; Lacasse y Mendelson, 2007). Aunque en este estudio el nivel de consumo no predijo la agresión psicológica en la pareja, futuros trabajos deberian investigar más exhaustivamente esta relación distinguiendo entre distintos tipos de agresión psicológica y diferentes grados en la severidad de la misma.

Son diversas las explicaciones teóricas que podrian dar cuenta de estos hallazgos. Además de los efectos farmacológicos directos del consumo de alcohol y otras drogas, es posible que el uso de sustancias y la violencia, junto con otros comportamientos de riesgo para la salud, formen parte de una constelación de conductas problemáticas en la adolescencia que compartan los mismos predictores individuales y ambientales (Feldstein y Miller, 2006). En consecuencia, consumo de sustancias y violencia en el noviazgo podrían constituir dos facetas de un patrón más generalizado de conductas de riesgo, lo cual es congruente con la Teoría de los Comportamientos Problema (Jessor et al., 2003). En esta línea, los individuos con altos niveles de consumo de sustancias podrían tender también a asociarse con otros iguales problemáticos, a implicarse actividades de búsqueda de sensaciones 0 a verse envueltos en un estilo de vida antisocial (Quigley y Leonard, 2000). Igualmente, la relación hallada entre consumo de sustancias y violencia podría ser de carácter bidireccional con influencias reciprocas, de forma tal que el uso de sustancias durante la adolescencia incremente la 
probabilidad de agresión y viceversa, tal y como se ha sugerido en otros trabajos (Abbey et al., 2004; Huang et al., 2001; Quigley y Leonard, 2000; White, Loeber, Stouthhamer-Loeber y Farrington, 1999). Esta relación podría verse modificada en el transcurso de la adolescencia, siendo de mayor magnitud entre los catorce y los quince años, lo cual apunta a la conveniencia de realizar intervenciones preventivas tempranas (White et al., 1999).

Independientemente de la direccionalidad o mecanismo de asociación, los hallazgos de este estudio indican que los adolescentes con un patrón de elevado consumo de alcohol y drogas ilegales se encuentran en una situación de vulnerabilidad para ejercer agresión física y sexual en sus relaciones de pareja. Futuros estudios deberian investigar la relación entre consumo de sustancias, violencia en el noviazgo y otros comportamientos de riesgo para la salud de los adolescentes, como las conductas sexuales de riesgo, el empleo de métodos inadecuados para el control del peso o el riesgo de embarazo no deseado.

Dos limitaciones del estudio deberían ser tenidas en cuenta al interpretar los resultados. La primera es que la muestra estuvo compuesta por adolescentes y jóvenes entre 15 y 20 años de centros públicos preuniversitarios. Por esta razón se debería ser precavido en la generalización de los hallazgos a otras poblaciones con características diferentes como, por ejemplo, los universitarios. En segundo lugar, el instrumento para medir el consumo de drogas ilegales muestreó únicamente el uso de marihuana y el de anfetaminas. Futuros trabajos deberían incluir también el consumo de otras sustancias cuyo uso es relativamente frecuente entre los jóvenes, tales como la cocaína.

Estos resultados tienen importantes implicaciones prácticas, ya que ponen de manifiesto la importancia de diseñar estrategias de prevención más holísticas que aborden varias, y no solo una, conductas de riesgo durante la adolescencia. Hasta el momento, la mayor parte de las intervenciones preventivas en nuestro país se han focalizado en comportamientos problemáticos específicos (p.ej., violencia, consumo de tabaco, alcohol, etc.). Sin embargo, la evidencia muestra que estos comportamientos tienden a coocurrir, indicando que los esfuerzos preventivos han de tener cuenta las interrelaciones entre múltiples actividades de riesgo (Zweig, 1999). Los programas de prevención que tomen en consideración diversas conductas problema en la adolescencia podrían ser más efectivos que aquellos otros focalizados únicamente en aspectos puntuales. $Y$, a tenor de los resultados obtenidos, ello parece especialmente importante de cara a la prevención, ya desde la adolescencia, de dos de los problemas sociales más acuciantes, el consumo de sustancias y la violencia en la pareja.

\section{REFERENCIAS}

Abbey, A., Zawacki, T., Buck, P. O., Clinton, A. M. y McAuslan, P. (2004). Sexual assault and alcohol consumption: What do we know about their relationship and what types of research are still needed? Aggression and Violent Behavior, 9, 271-303.

Abbey, A., Zawacki, T. y McAuslan, P. (2000). Alcohol's effects on sexual perception. Journal of Studies on Alcohol, 61, 688-697.

Ackard, D. M. y Neumark-Sztainer, D. (2002). Date violence and date rape among adolescents: Associations with disordered eating behaviors and psychological health. Child Abuse \& Neglect, 26, 455-473.

Calvete, E. y Estévez, A. (2009). Consumo de drogas en adolescentes: El papel del estrés, la impulsividad y los esquemas relacionados con la falta de límites. Adicciones, 21, 49-56.

Cascardi, M., Avery-Leaf, S., O'Leary, K. D. y Slep, A. M. S. (1999). Factor structure and convergent validity of the conflict tactics scale in high school students. Psychological Assessment, 11, 546-555.

Champion, H. L. O., Foley, K. L., DuRant, R. H., Hensberry, R., Altman, D. y Wolfson, M. (2004). Adolescent sexual victimization, use of alcohol and other substances, and other health risk behaviors. Journal of Adolescent Health, 35, 321-328.

Crowne, D. P. y Marlowe, D. (1960). A new scale of social desirability independent of psychopathology. Journal of Consulting Psychology, 24, 349-354.

Dobash, R. P. y Dobash, R. E. (2004). Women's violence to men in intimate relationships: Working on a puzzle. British Journal of Criminology, 44, 324-349.

DuRant, R., Champion, H., Wolfson, M., Omli, M., McCoy, T., D'Agostino, R. B., Jr., et al. (2007). Date fighting experiences among college students: Are they associated with other health-risk behaviors? Journal of American College Health, 55, 291-296.

Eaton, D. K., Davis, K. S., Barrios, L., Brener, N. D. y Noonan, R. K. (2007). Associations of dating violence victimization with lifetime participation, co-occurrence, and early initiation of risk behaviors among U.S. high school students. Journal of Interpersonal Violence, 22, 585-602.

Eisner, M. (2002). Crime, problem drinking, and drug use: Patterns of problem behavior in cross-national perspective. Annals of the American Academy of Political and Social Science. Special Issue: Early Adulthood in Cross-National Perspective, 580, 201-225.

Estudes (2008). Informe de la encuesta estatal sobe el uso de drogas en estudiantes de enseñanzas secundarias. Madrid: Delegación del Gobierno para el Plan Nacional sobre Drogas.

Feldstein, S. W. y Miller, W. R. (2006). Substance use and risk-taking among adolescents. Journal of Mental Health. Special Issue: New Advancements in the Study of Co-Occurring Substance use and Psychiatric Disorders, 15, 633-643.

Ferrando, P. J. y Chico, E. (2000). Adaptación y análisis psicométrico de la escala de deseabilidad social de Marlowe y Crowne. Psicothema, 12, 383-389.

Foshee, V. A. (1996). Gender differences in adolescent dating abuse prevalence, types and injuries. Health Education Research, 11, 275-286.

Foshee, V. A., Linder, F., MacDougall, J. E. y Bangdiwala, S. (2001). Gender differences in the longitudinal predictors of adolescent dating violence. Preventive Medicine: An International Journal Devoted to Practice and Theory, 32, 128-141. 
Harrison, L. D., Erickson, P. G., Adlaf, E. y Freeman, C. (2001). The drugs-violence nexus among American and Canadian youth. Substance Use \& Misuse, 36, 2065-2086.

Hickman, L. J., Jaycox, L. H. y Aronoff, J. (2004). Dating violence among adolescents: Prevalence, gender distribution, and prevention program effectiveness. Trauma, Violence, \& Abuse, 5, 123-142.

Hines, D. A. y Saudino, K. J. (2003). Gender differences in psychological, physical, and sexual aggression among college students using the Revised Conflict Tactics Scales. Violence and Victims, 18, 197-217.

Hines, D. A. y Straus, M. A. (2007). Binge drinking and violence against dating partners: The mediating effect of antisocial traits and behaviors in a multinational perspective. Aggressive Behavior, 33, 441-457.

Howard, D. E., Wang, M. 0. y Yan, F. (2007). Psychological factors associated with reports of physical dating violence among U.S. adolescent females. Adolescence, 42, 311-324.

Huang, B., White, H. R., Kosterman, R., Catalano, R. F. y Hawkins, J. D. (2001). Developmental associations between alcohol and interpersonal aggression during adolescence. Journal of Research in Crime and Delinquency, 38, 64-83.

Ito, T. A., Miller, N. y Pollock, V. E. (1996). Alcohol and aggression: A meta-analysis on the moderating effects of inhibitory cues, triggering events, and self-focused attention. Psychological Bulletin, 120, 60-82.

Jessor, R. (1987). Problem-behavior theory, psychosocial development, and adolescent problem drinking. British Journal of Addiction. Special Issue: Psychology and Addiction, 82, 331-342.

Jessor, R. (1991). Risk behavior in adolescence: A psychosocial framework for understanding and action. Journal of Adolescent Health. Special Issue: Adolescents at Risk, 12, 597-605.

Jessor, R. (1993). Successful adolescent development among youth in high-risk settings. American Psychologist. Special Issue: Adolescence, 48, 117-126.

Jessor, R., Turbin, M. S., Costa, F. M., Dong, O., Zhang, H. y Wang, C. (2003). Adolescent problem behavior in China and the United States: A cross-national study of psychosocial protective factors. Journal of Research on Adolescence, 13, 329-360.

Jiménez-Muro, A., Beamonte, A., Marqueta, A., Gargallo, P., Nerin, I. (2009). Consumo de drogas en estudiantes universitarios de primer curso. Adicciones, 21, 21- 28.

Kaura, S. A. y Lohman, B. J. (2007). Dating violence victimization, relationship satisfaction, mental health problems, and acceptability of violence: A comparison of men and women. Journal of Family Violence, 22, 367-381.

King, G., Flisher, A. J., Noubary, F., Reece, R., Marais, A. y Lombard, C. (2004). Substance abuse and behavioral correlates of sexual assault among South African adolescents. Child Abuse \&t Neglect, 28, 683-696.

Kuntsche, E., Knibbe, R., Engels, R. y Gmel, G. (2007). Bullying and fighting among adolescents--do drinking motives and alcohol use matter? Addictive Behaviors, 32, 3131-3135.

Lacasse, A. y Mendelson, M. J. (2007). Sexual coercion among adolescents: Victims and perpetrators. Journal of Interpersonal Violence, 22, 424-437.

MacDonald, J. M., Piquero, A. R., Valois, R. F. y Zullig, K. J. (2005). The relationship between life satisfaction, risk-taking behaviors, and youth violence. Journal of Interpersonal Violence, 20, 14951518.
Muñoz-Rivas, M. J., Andreu , J. M., Graña, J. L. G., O'Leary, D. K. y González, M. (2007). Validación de la versión modificada de la Conflicts Tactics Scale (M-CTS) en población juvenil española. Psicothema, 19, 693-698.

Muñoz-Rivas, M. J., Graña, J. L., O'Leary, K. D. y González, P. G. (2007). Agresión física y psicológica en las relaciones de noviazgo en universitarios españoles. Psicothema, 19, 102-107.

Muñoz-Rivas, M. J., Graña, J. L., O'Leary, K. D. y González, P. G. (2009). Prevalence and predictors of sexual aggression in dating relationships of adolescents and young adults. Psicothema, 21, $234-240$.

O'Leary, K. D. y Cascardi, M. (1998). Physical aggression in marriage: A developmental analysis. New York, NY, US: Cambridge University Press.

O'Leary, K. D., Slep, A. M. S., Avery-Leaf, S. y Cascardi, M. (2008). Gender differences in dating aggression among multiethnic high school students. Journal of Adolescent Health, 42, 473-479.

Peña, M. E., Andreu, J. M. y Graña, J. L. (2008). Multivariate model of antisocial behavior and substance use in Spanish adolescents. Journal of Child and Adolescent Substance Abuse.

Poitras, M. y Lavoie, F. (1995). A study of the prevalence of sexual coercion in adolescent heterosexual dating relationships in a Quebec sample. Violence and Victims, 10, 299-313.

Quigley, B. M. y Leonard, K. E. (2000). Alcohol, drugs, and violence. Needham Heights, MA, US: Allyn \& Bacon.

Schumacher, J. A. y Slep, A. M. S. (2004). Attitudes and dating aggression: A cognitive dissonance approach. Prevention Science, 5, 231-243.

Shook, N. J., Gerrity, D. A., Jurich, J. y Segrist, A. E. (2000). Courtship violence among college students: A comparison of verbally and physically abusive couples. Journal of Family Violence, 15, 1-22.

Silverman, J. G., Raj, A., Mucci, L. A. y Hathaway, J. E. (2001). Dating violence against adolescent girls and associated substance use, unhealthy weight control, sexual risk behavior, pregnancy, and suicidality. JAMA: Journal of the American Medical Association, 286, 572-579.

Straus, M. A., Hamby, S. L., Boney-McCoy, S. y Sugarman, D. B. (1996). The revised Conflict Tactics Scales (CTS2): Development and preliminary psychometric data. Journal of Family Issues, 17, 283-316.

Swahn, M. H., Bossarte, R. M. y Sullivent, E. E. (2008). Age of alcohol use initiation, suicidal behavior, and peer and dating violence victimization and perpetration among high-risk, seventh-grade adolescents. Pediatrics, 121, 297-305.

White, H. R., Loeber, R., Stouthhamer-Loeber, M. y Farrington, D. P. (1999). Developmental associations between substance use and violence. Development and psychopathology, 11, 785-803.

White, J. W., Merrill, L. L. y Koss, M. P. (2001). Predictors of premilitary courtship violence in a navy recruit sample. Journal of Interpersonal Violence, 16, 910-927.

Zweig, J. (1999). A longitudinal examination of the consequences of sexual victimization for rural young adult women. The Journal of Sex Research, 36, 396. 
